\title{
Comparison of Musculoskeletal Disorders in School Going Children Before and After COVID-19
}

\author{
Neha P Patel ${ }^{1}$, Megha S Sheth ${ }^{2}$ \\ ${ }^{1}$ Postgraduate Physiotherapy Student, SBB College of Physiotherapy, Ahmedabad, Gujarat, India \\ ${ }^{2}$ Lecturer, SBB College of Physiotherapy, Ahmedabad, Gujarat, India \\ Corresponding Author: Neha P Patel
}

\begin{abstract}
Background: An outbreak of corona virus (COVID-19) disease causing severe acute respiratory syndrome first occurred in China. About 107 countries implemented school closures. This lockdown/quarantine was associated with changes in the daily activity among children, where less physical activity and more use of electronic devices was seen. The study aimed to compare musculoskeletal disorders among children before and after COVID-19.

Methodology: One hundred thirty one school-going children, selected by convenience sampling, aged 6-18 years, studying online since at least one month, participated in this cross-sectional study. The participants filled a self-designed, close-ended questionnaire of 24 items. Descriptive analysis was done using Microsoft excel.

Results: $80 \%$ children (average age 13.7 years) used electronic devices less than 2 hours for study, 90\% for games, social media and communication before COVID-19. After COVID-19, 93\%children used electronic devices more than 2 hours for study, $72 \%$ children used electronic devices less than 2 hours for games, social media and communication.51\% children had musculoskeletal pain after COVID-19. Children with pain in the neck (39\%), back (15\%), neck and back (12\%), right or left shoulder $(6 \%)$, both shoulders $(6 \%)$ and more than one part involved $(21 \%)$. Neck pain was mild $(28 \%)$, moderate $(65 \%)$, severe $(6.7 \%)$. Severity of back pain was mild $(55 \%)$, moderate $(32 \%)$, severe (14\%). Severity of shoulder pain seen was mild $(75 \%)$, moderate $(26 \%)$.Severity of arm pain was mild (84\%), moderate (17\%). 52\% Children were exercising before COVID-19, $60 \%$ after COVID-19.

Conclusion: After COVID-19 there is an increase in the prevalence of musculoskeletal disorders (neck, back, shoulder and arm pain) among children with an increase in the time of use of electronic devices.
\end{abstract}

Keywords: Musculoskeletal disorders, School going children, Covid-19, Electronic devices

\section{INTRODUCTION}

At the end of 2019, a novel corona virus was identified as the cause of a cluster of pneumonia cases in Wuhan, a city in the Hubei Province of China. It rapidly spread, resulting in an epidemic throughout China, followed by an increasing number of cases in other countries throughout the world. In February 2020, the World Health Organization designated the disease COVID-19, which stands for corona virus disease 2019. The virus that causes COVID-
19 is designated severe acute respiratory syndrome corona virus 2 (SARS-CoV-2); previously, it was referred to as $2019-\mathrm{nCoV}$ [1]. During this pandemic, many countries implemented a lock down and 107 countries implemented school closures too ${ }^{[2]}$.

Musculoskeletal Disorders (MSD) are defined as "conditions that affect the loco-motor system; that is, muscles, bones, joints and associated tissues such as tendons and ligaments, as listed in the International Classification of Diseases" (WHO, 2019) ${ }^{[3]}$ 
Over the last few years, there is almost a $25 \%$ increase in the number of people suffering from MSD around the world ${ }^{[3]}$. Musculoskeletal conditions are usually characterized by pain, mobility restrictions, lack of skills and decreased functional ability. It also reduces people's ability to work and cooperate in social roles. Most people with MSD suffer back, neck, and shoulder pain ${ }^{[4]}$.

This lockdown or quarantine is associated with changes in the daily activity routine among all sectors of population in general. The corona virus (COVID-19) pandemic makes it challenging to maintain a physically active lifestyle ${ }^{[5]}$. Less physical activity has become a habit, and more use of electronic device for different purposes has exceeded all previous limits ${ }^{[6]}$.

Electronic devices include mobile, laptop, camera, watch, tablet and computer among others. Devices like mobile, laptop, tablet and computer can be used for teaching-learning purposes. Because of the need to stay at home, people of all ages have been seen to spend more time with these devices. The same is seen with children, mainly school going children who continued their education remotely online and may have predisposed children to MSD.

Studies on children with MSD's are few. Aim of the study was to compare the prevalence of musculoskeletal disorders in children before and after COVID-19.

\section{METHOD}

An observational study was conducted from September 2020 to October 2020. One hundred thirty one school-going children were selected by convenience sampling. A Google self-designed questionnaire was generated which was given to three physiotherapists who approved of the content of the questionnaire with few modifications. Questionnaires were given to five children for addition, deletion or modification of questions before circulating it among school going children. After final approval of the content of questions by authors, the questionnaires were circulated via what's app. The Questionnaire took 5-10 minutes to complete.

Boys and girls, 6 to 18 years old school going children who were studying online at present since at least one month were included. Children not studying online at present were excluded.

Questionnaire consisted of 24 items, which included name, age, gender, standard, handedness, sleeping hours and questions related to electronic devices used, before and after COVID-19. It also included questions on musculoskeletal pain present in neck, shoulder, arm, back and severity of pain as mild, moderate, and severe. Time spent exercising and types of exercise before and after COVID-19 were also asked. Descriptive analysis was done in Microsoft excel.

\section{RESULTS}

One hundred thirty one children participated in the study, 63(48.1\%) were boys and $68(51.9 \%)$ were girls. The mean age was 13.7 years $(\mathrm{SD}=3.05)$. Right handed children were 109(83.2\%), left handed were $17(13 \%)$ and children who could use both hands were $5(3.8 \%)$. Number of children studying in which year is shown in Table1.

Table: 1 Number of children studying in which year

\begin{tabular}{|l|l|}
\hline standard in which studying & Frequency \\
\hline $1^{\text {st }}$ & 2 \\
\hline $2^{\text {nd }}$ & 3 \\
\hline $3^{\text {rd }}$ & 7 \\
\hline $4^{\text {th }}$ & 9 \\
\hline $5^{\text {th }}$ & 7 \\
\hline $6^{\text {th }}$ & 6 \\
\hline $7^{\text {th }}$ & 11 \\
\hline $8^{\text {th }}$ & 12 \\
\hline $9^{\text {th }}$ & 13 \\
\hline $10^{\text {th }}$ & 13 \\
\hline $11^{\text {th }}$ & 6 \\
\hline $12^{\text {th }}$ & 42 \\
\hline Total & $\mathbf{1 3 1}$ \\
\hline
\end{tabular}

Different positions used when children used electronic devices are shown in graph 1. Sleeping hours after COVID-19 is shown in graph 2. Different types of electronic devices used by children for study, games, social media and communication is shown in graph 3. Graphs 
Neha P Patel et.al. Comparison of musculoskeletal disorders in school going children before and after COVID19.

4, 5 show the use of electronic devices for study, game, social media and communication before and after COVID-19 (in hours).

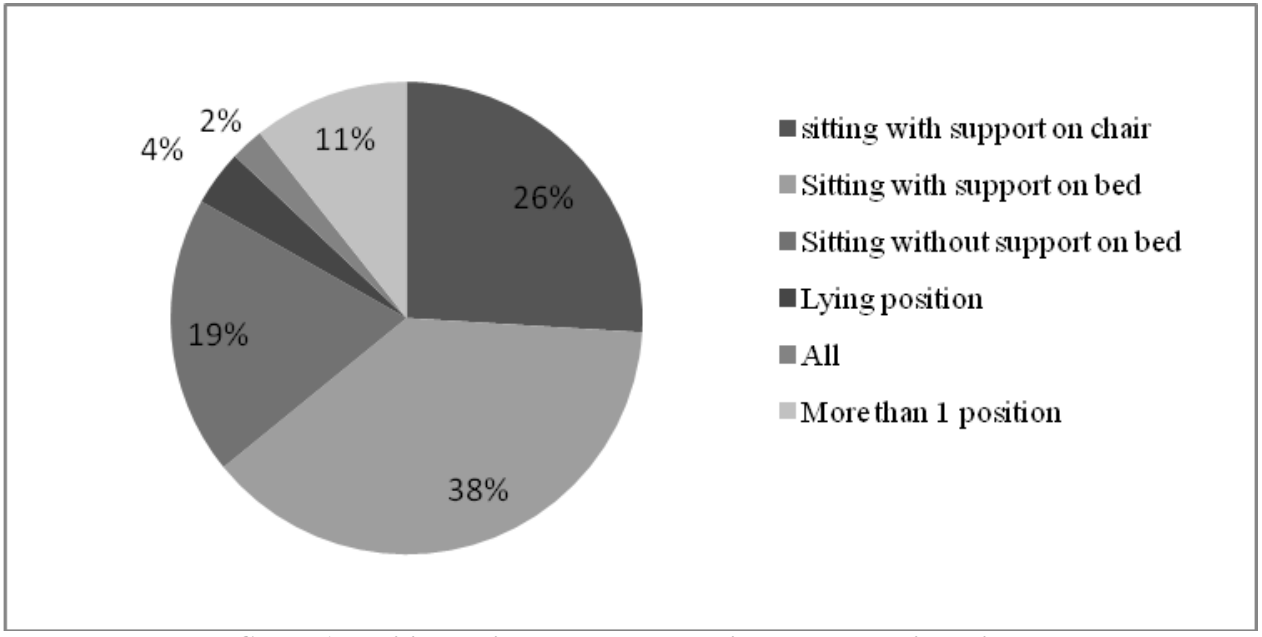

Graph 1: Positions children have when using the electronic devices

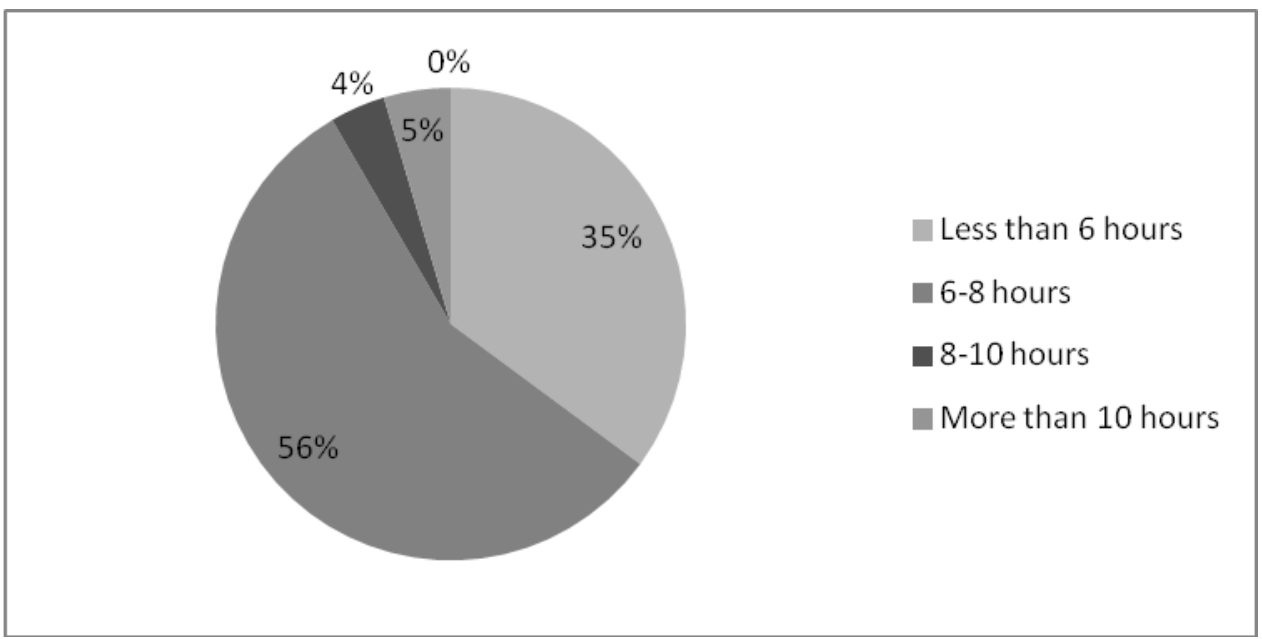

Graph 2: Sleeping hours after COVID-19

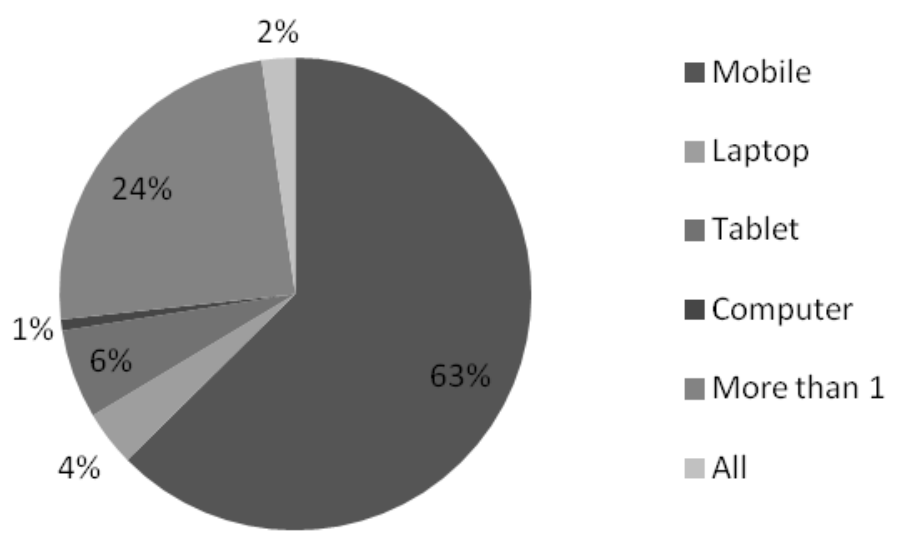

Graph 3: Type of electronic devices used by children

$51 \%$ children had musculoskeletal disorders after COVID-19. Location of pain is shown in graph 6. Severity of pain is shown in graph $7.52 \%$ children were doing exercises before COVID-19 when 61\% children were doing exercises after COVID19. Duration of exercises is shown in graph 
Neha P Patel et.al. Comparison of musculoskeletal disorders in school going children before and after COVID19.

8 and type of exercises are shown in graph 9.

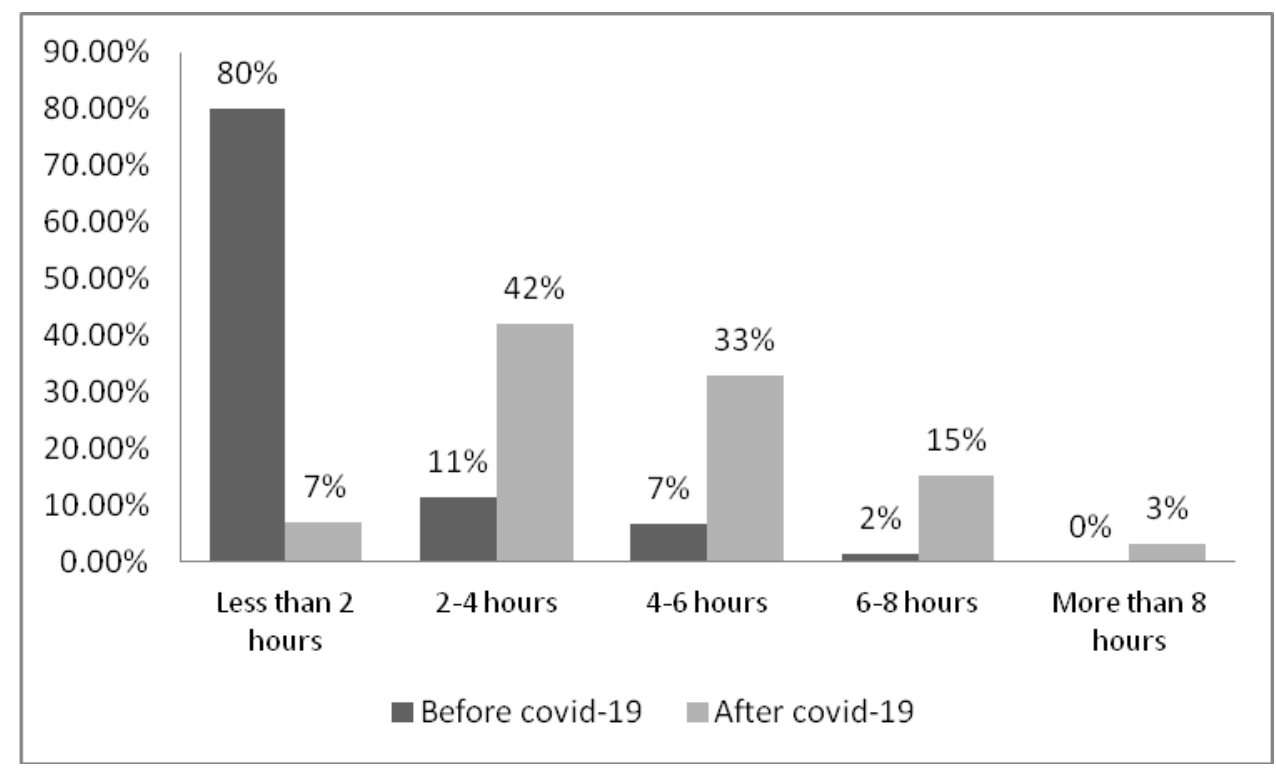

Graph 4: Use of electronic devices for study before and after COVID-19 (in hours)

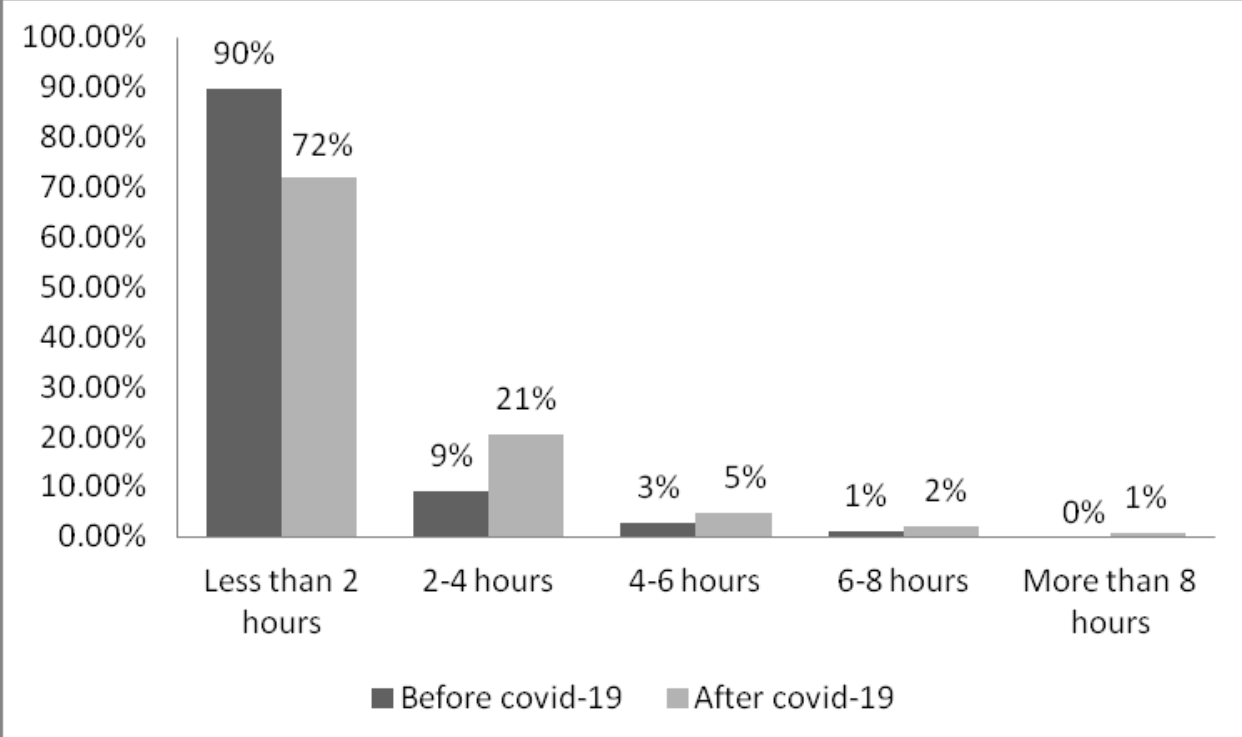

Graph 5: Use of electronic devices for games, social media and communication before and after COVID-19(in hours)

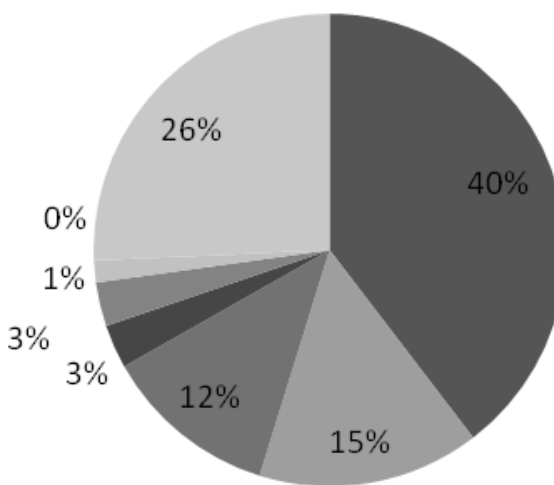

neck

Back

Neck+Back

- Right shoulder

- Left shoulder

Right arm

- Left arm

More than 1 part involved

Graph 6: Location of pain 
Neha P Patel et.al. Comparison of musculoskeletal disorders in school going children before and after COVID19.

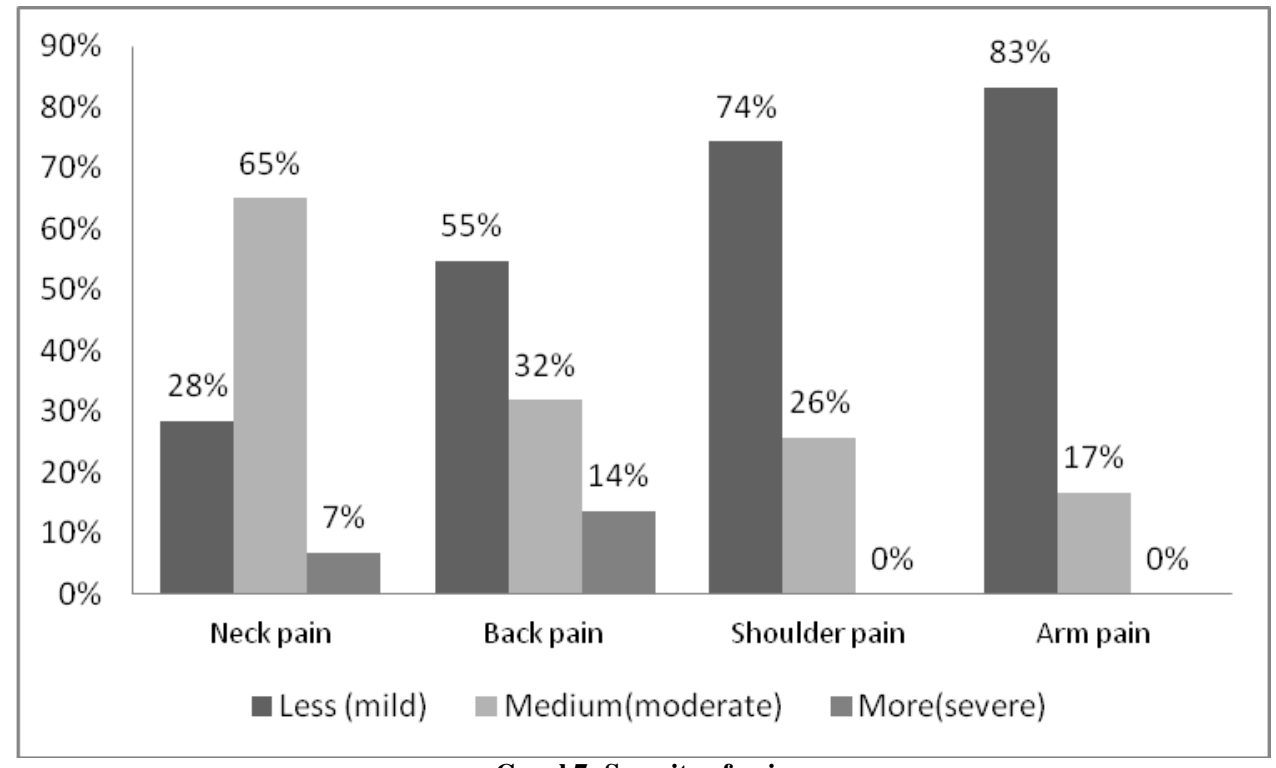

Graph7: Severity of pain

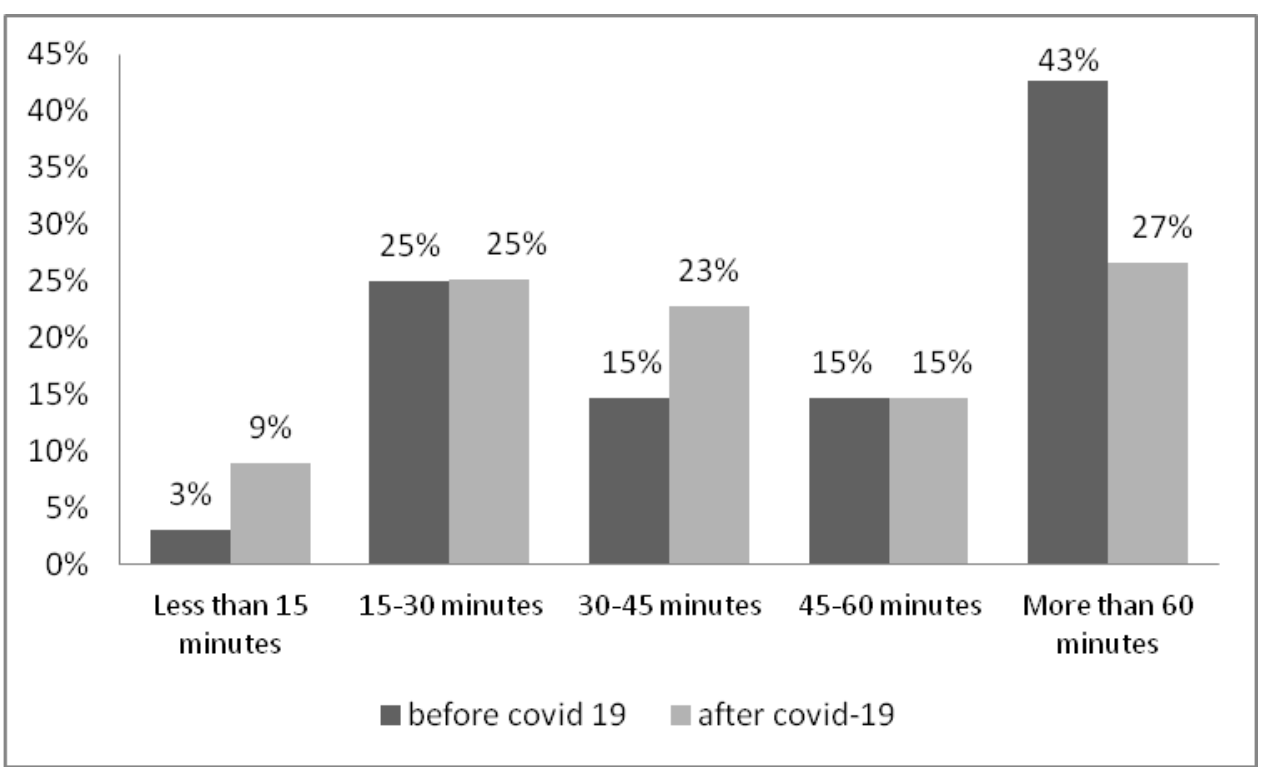

Graph 8 : Duration of exercise before and after covid 19

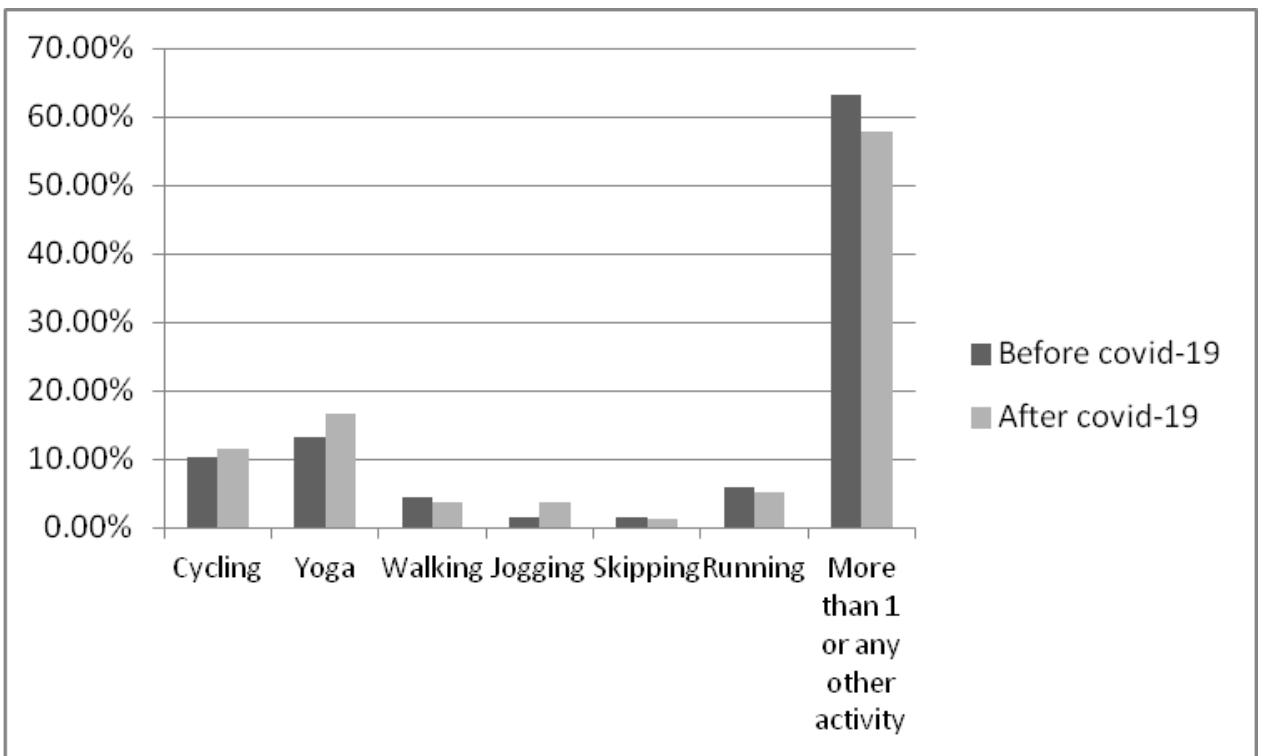

Graph9: Type of exercise before and after covid-19 


\section{DISCUSSION}

$80 \%$ children (average age 13.7 years) used electronic devices less than 2 hours for study, $90 \%$ for games, social media and communication before COVID19.After COVID-19, $93 \%$ children used electronic devices more than 2 hours for study, $72 \%$ children used electronic devices less than 2 hours for games, social media and communication. $51 \%$ children had musculoskeletal pain after COVID-19. Children with pain in the neck (39\%), back $(15 \%)$, neck and back (12\%), right or left shoulder $(6 \%)$, both shoulders $(6 \%)$ and more than one part involved $(21 \%)$. Neck pain was mild (28\%), moderate (65\%), severe $(7 \%)$. Severity of back pain was mild (55\%), moderate (32\%), severe (14\%). Severity of shoulder pain seen was mild (75\%), moderate (26\%). Severity of arm pain was mild (84\%), moderate (17\%). 52\% Children were exercising before COVID-19, $60 \%$ after COVID-19.

The present study shows that after COVID-19 use of electronic devices for study hours has increased compared to before COVID-19. During this pandemic situation play-ground activity of the children has stopped. They are also not able to meet their peer group. So, to stay in contact with his/her friend and for playing games children use electronic devices. So duration of use of electronic devices has increased. The present study found that after COVID-19 the use of electronic devices for social media, games and communication hours has increased. This is similar to findings of Amro A et al who showed in their study that quarantine increased the time of use of social media, which in turn increased the prevalence and severity of MSD among university students [6] Similarly Rimba et al. showed that the excessive time of use on social media leads to more hours with stress on posture, and that the vast majority did not care of considering postural recommendations while using social media. There was statistically significant correlation between the extent of this consideration and the severity of the MSD ${ }^{[7]}$.

This study showed $51 \%$ children had musculoskeletal pain (neck, back, arm and hand) present after COVID-19. More children had pain over neck, back or combined neck and back pain present. Lesser children presented with shoulder and arm pain. Moderate intensity pain was present in neck and back. Mild intensity pain was present in shoulder and arm. Amro A, et al showed in their study an increase of severity of musculoskeletal disorders (MSD) as measured by a scale of 0-10 after the quarantine in terms of severity of headache, neck and back pain ${ }^{[6]}$.

After COVID-19 exercising level was found to be increased in children. Duration of exercise may or may not be different before and after COVID-19. Yoga (type of exercise) as an activity was increased in after COVID-19.Exercise is required for physical and mental balance. During the pandemic situation children could not perform outdoor activities. Also continued use for electronic devices for study, games, communication and social media was seen. So to maintain physical and mental health in children more activity and exercise was seen. Amatriain-Fernández, et al showed that physical activity and physical exercise might be key factors to help the population to better tolerate pandemic periods at both the mental and physical levels ${ }^{[8]}$.

This study showed $56 \%$ children were sleeping 6-8 hours a day, after COVID-19. Sayed A Tantawy showed a negative correlation between night sleeping hours and severity of MSD reported by student ${ }^{[9]}$. Their study showed factors affecting the duration of sleep included the amount of homework, extracurricular activities, social activities, electronics used and sports. The factors were however not assessed in the present study.

A few limitations of the study were that only school going children who used electronic devices for minimum 1 hour for study were included. Before COVID-19, 

19.

pain was not assessed. Before COVID-19, sleeping hours not asked. Statistical tests for comparison of before and after COVID -19 were not applied. Correlation between exercise and MSD and use of electronic devices and MSD was not done. Future studies can be conducted to find correlation between exercise and MSD and use of electronic devices and MSD. Intelligent use of electronic devices can be taught to children and the effect of that on the pain can be studied.

\section{CONCLUSION}

After COVID-19 there is an increase in the time of use of electronic device and an increase in the prevalence of musculoskeletal disorders (neck, back, shoulder and arm pain) among school-going children.

\section{Acknowledgements}

We are thankful to all participants who helped to complete this study.

\section{Conflict of Interest: None}

\section{Source of Funding: None}

\section{Ethical Approval: Approved}

\section{REFERENCES}

1. McIntosh K, Hirsch MS, Bloom A. Coronavirus disease 2019 (COVID-19): Epidemiology, virology, and prevention. Lancet. Infect. Dis. 2020 Jul;1:2019-20.

2. Viner RM, Russell SJ, Croker H, Packer J, Ward J, Stansfield C, Mytton O, Bonell C, Booy R. School closure and management practices during coronavirus outbreaks including COVID-19: a rapid systematic review. The Lancet Child \& Adolescent Health. 2020 May 1;4(5):397-404
3. https://www.who.int/news-room/factsheets/detail/musculoskeletal-conditions

4. Yang G, Cao J, Li Y, Cheng P, Liu B, Hao Z, Yao H, Shi D, Peng L, Guo L, Ren Z. Association between internet addiction and the risk of musculoskeletal pain in Chinese college freshmen-a cross-sectional study. Frontiers in psychology. 2019 Sep 3;10:1959.

5. Shariat A, Hakakzadeh A, Cleland J. Homebased exercise note in Covid-19 quarantine situation for office workers: A commentary. Work. 2020 Jun 16(Preprint):1-2.

6. Amro A, Albakry S, Jaradat M, Khaleel M, Kharroubi T, Dabbas A, Dwaik R. Musculoskeletal Disorders and Association with Social Media Use Among University Students at the Quarantine Time Of COVID-19 Outbreak.

7. Rimba JT, Naiem F, Rahim MR. Relationship between work posture and musculoskeletal disorders (Msds) at processing workers in Pttoarco Jaya, Rantepao city year 2017. SCOPUS IJPHRD CITATION SCORE. 2019 Jul 1;10(7):1025.

8. Wegner M, Amatriain-Fernández S, Kaulitzky A, Murillo-Rodriguez E, Machado S, Budde H. Systematic review of meta-analyses: Exercise effects on depression in children and adolescents. Frontiers in psychiatry. 2020 Mar 6;11:81.

9. Topping G. Students Are Not Sleeping Enough: Comparing High School Sleep Schedules to College Sleep Schedules-A Look into the Importance of Sleep, the Factors Affecting Sufficient Sleep and its Effect on Health and Academic Success. https://digitalcommons.ithaca.edu/whalen/2 019/posters/186/

How to cite this article: Patel NP, Sheth MS. Comparison of musculoskeletal disorders in school going children before and after COVID19. Int J Health Sci Res. 2021; 11(5): 67-73. DOI: https://doi.org/10.52403/ijhsr.20210510 